\title{
Assessment of the most effective method of teaching cricoid pressure force
}

\author{
Jasmine Whitaker ${ }^{1}$, Natalie Elsdon ${ }^{2}$, John Moran ${ }^{3}$, Jonathan Hulme ${ }^{4}$, and Sandra Peake ${ }^{3}$ \\ ${ }^{1}$ Royal Gwent Hospital \\ ${ }^{2}$ University of Birmingham Birmingham Medical School \\ ${ }^{3}$ The Queen Elizabeth Hospital \\ ${ }^{4}$ Sandwell and West Birmingham Hospitals NHS Trust
}

October 27, 2020

\begin{abstract}
Cricoid pressure is used to reduce the risk of aspiration during rapid sequence induction. The recommended force applied to the cricoid is $10-20$ newtons $(\mathrm{N} ; 1.020-1.040 \mathrm{~kg})$ on awake patients and $30-40 \mathrm{~N}$ (3.060-4.080 kg) on anaesthetised patients. However clinically, it is difficult to estimate the required force. We assessed the effectiveness of 3 recommended teaching methods on the ability to apply the correct force using an airway model that simulated "awake" and "anaesthetised" patients. Thirty nurses and doctors from two hospitals and with clinical experience applying cricoid pressure were included. Measurements of baseline force for "awake" and "anaesthetised" patients were obtained from all participants using measuring scales. Participants were blinded to the force applied. Participants were taught one of three different techniques: biofeedback, nose and syringe. Post-teaching, blinded force measurements were repeated. Data analysis was performed using a linear mixed model and marginal prediction models of applied force reported. For "awake" patients, nose method forces were within the recommended range (mean 14.6N, 95\%CI 9.7-19.4). The biofeedback method led to predicted forces at the upper limit of recommended (21.6N, 95\%CI 16.7-26.4) and the syringe method forces were greater than recommended (29.0N, 95\%CI 23.9-34.0). For "anaesthetised" patients, nose method forces were less than recommended $(26.3 \mathrm{~N}, 95 \% \mathrm{CI} 21.6-31.1)$, the biofeedback method led to predicted forces within range (33.4N, 28.4-38.3) and syringe method forces were above those recommended (40.8N, 95\% CI 35.8-45.8). The biofeedback technique is the most effective method for teaching the application of recommended cricoid pressure force for both awake and anaesthetised patients.
\end{abstract}

\section{Original research}

\section{ASSESSMENT OF THE MOST EFFECTIVE METHOD OF TEACHING CRICOID PRES- SURE FORCE}

Short title: The most effective method of teaching cricoid pressure force

Jasmine Whitaker, ${ }^{1}$ Natalie Elsdon ${ }^{2}$, John Moran ${ }^{3}$, Jonathan Hulme ${ }^{4}$ and Sandra Peake ${ }^{5}$

1 Anaesthetics Core Trainee, Royal Gwent Hospital, Newport, United Kingdom, 2 Medical student, University of Birmingham Medical School, Birmingham, United Kingdom, 3 Associate Professor and Critical Care Consultant, The Queen Elizabeth Hospital, Adelaide, Australia, 4 Consultant in Anaesthesia and Intensive Care Medicine, Sandwell and West Birmingham NHS Trust, Birmingham, United Kingdom, 6 Professor and Critical Care Consultant, The Queen Elizabeth Hospital, Adelaide, Australia

Submitting and corresponding author: Jasmine Whitaker

Department of Anaesthesia, Royal Gwent Hospital, Cardiff Rd, Newport NP20 2UB, United Kingdom. 
Email: jjmwhitaker@gmail.com, Telephone: 07828270568.

* Presented as oral presentation at the World Congress of Intensive Care 2019, Melbourne Convention and Exhibition Centre Melbourne, Australia, 14-18 October 2019

Presented as poster presentation at The Combined New Zealand Anaesthesia Annual Scientific Meeting + Annual Queenstown Update in Anaesthesia (AQUA) 2019, Millennium \& Copthorne Hotels, Queenstown, New Zealand, 21 - Saturday 24 August 2019.

The author's institutional affiliations where the work was carried out: City and Sandwell Hospitals, Birmingham

The data that support the findings of this study are available from the corresponding author upon reasonable request.

Keywords: cricoid pressure; rapid sequence induction; teaching methods

\section{SUMMARY}

Cricoid pressure is used to reduce the risk of aspiration during rapid sequence induction. The recommended force applied to the cricoid is 10-20 newtons $(\mathrm{N} ; 1.020-1.040 \mathrm{~kg})$ on awake patients and $30-40 \mathrm{~N}(3.060-4.080$ $\mathrm{kg}$ ) on anaesthetised patients. However clinically, it is difficult to estimate the required force. We assessed the effectiveness of 3 recommended teaching methods on the ability to apply the correct force using an airway model that simulated "awake" and "anaesthetised" patients.

Thirty nurses and doctors from two hospitals and with clinical experience applying cricoid pressure were included. Measurements of baseline force for "awake" and "anaesthetised" patients were obtained from all participants using measuring scales. Participants were blinded to the force applied. Participants were taught one of three different techniques: biofeedback, nose and syringe. Post-teaching, blinded force measurements were repeated. Data analysis was performed using a linear mixed model and marginal prediction models of applied force reported.

For "awake" patients, nose method forces were within the recommended range (mean 14.6N, 95\%CI 9.719.4). The biofeedback method led to predicted forces at the upper limit of recommended $(21.6 \mathrm{~N}, 95 \% \mathrm{CI}$ 16.7-26.4) and the syringe method forces were greater than recommended (29.0N, 95\%CI 23.9-34.0). For "anaesthetised" patients, nose method forces were less than recommended (26.3N, 95\%CI 21.6-31.1), the biofeedback method led to predicted forces within range $(33.4 \mathrm{~N}, 28.4-38.3)$ and syringe method forces were above those recommended $(40.8 \mathrm{~N}, 95 \%$ CI $35.8-45.8)$.

The biofeedback technique is the most effective method for teaching the application of recommended cricoid pressure force for both awake and anaesthetised patients.

Cricoid pressure, first described by Sellick in 1961, is used to reduce the risk of aspiration during induction by posterior displacement of the cricoid cartilage ring and compressing the oesophagus. Sellick recommended that cricoid pressure should be applied "lightly" whilst a patient is awake and "firm" when they are unconscious[1].

Several caveats are relevant to the application of cricoid pressure; one must be able to locate the cricoid cartilage correctly, know when to apply the pressure, know the direction that the pressure is applied and apply the correct force when the patient is awake and anaesthetised[2]. This study focuses on the cricoid pressure force and does not discuss these other aspects further.

Recommended force applied during cricoid pressure varies amongst the literature but is generally 10-20N $(1.020-1.040 \mathrm{~kg})$ on awake patients and $30-40 \mathrm{~N}(3.060-4.080 \mathrm{~kg})$ on anaesthetised patients[3-10]; however, it is difficult for staff to estimate this required force accurately in clinical practice[9,11].

For awake patients, a force greater than $10 \mathrm{~N}$ is required to prevent aspiration, whilst a force greater than $20 \mathrm{~N}$ can cause pain and retching. For anaesthetised patients, a force greater than $30 \mathrm{~N}$ is required to prevent 
aspiration, whereas a force greater than $40 \mathrm{~N}$ can cause trauma to the larynx[9]. Studies suggest that most assistants apply less cricoid pressure than is required during intubation[4,12-14].

Multiple methods have been described in the literature to teach the required cricoid pressure force. The most common methods include biofeedback[2,10,15-19], nose[7], and syringe[8,20]. In brief, biofeedback methods include an airway model connected to measuring scales and participants use real-time feedback to apply the recommended force to the model[2,10,15-19]. For the nose method, participants are instructed to use sufficient pressure to cause pain if applied to the bridge of the nose for anaesthetised patients[7]. The syringe method involves pressing the plunger of a $50 \mathrm{ml}$ closed syringe to specified depths, which are equivalent to the recommended forces for awake and anaesthetised patients[8,20].

Whilst most studies have reported the biofeedback and syringe methods to be effective in teaching the required force of cricoid pressure[8,17,20], there is limited research evaluating the nose method; albeit Escott et $a$ l.[7] reported that the nose method did not appear useful in teaching cricoid pressure force.

The aim of our study was to evaluate the comparative effectiveness of the biofeedback, nose and syringe methods of teaching the recommended cricoid pressure force on "awake" and "anaesthetised" patients using an airway model.

\section{METHODS}

\section{Population}

The study was conducted over one working day at the City and Sandwell Hospitals in the West Midlands region of the United Kingdom. Thirty Intensive Care Unit (ICU) and operating theatre (OT) doctors and nurses who assist or carry out rapid sequence induction (RSI) as part of their job role were eligible to participate and recruitment was according to convenience sampling and direct approach (Figure 4). Information on job roles and previous experience of applying cricoid pressure was collected. All participants were required to have previously carried out cricoid pressure on a patient.

\section{Baseline force measurement}

Baseline measurements of cricoid pressure force were obtained using a pharyngeal model ${ }^{\mathrm{TM}}$ (Laerdal, Sentrum, Stavanger, Norway) attached to measuring scales (Salter Colorweigh Electronic Kitchen Scale, China) (Figure 1). The maximum recording value of the scales was $6.0 \mathrm{~kg}$. Participants were instructed to press down on the airway model and indicate to the study researcher (JW) when the intended force was reached and maintained. Participants were blinded as to the applied force and measurement to the nearest $100 \mathrm{~g}$ was recorded by the study researcher (JW). Participants were blinded as to the applied force. Force measurements were recorded for all participants on "awake" and "anaesthetised" patients, with 3 attempts for each. Participants were required to remove their hand from the model between measurements of applied force.

\section{Intervention}

Thirty participants were sequentially allocated in blocks of 3 to the biofeedback $(n=10)$, nose analogy $(n=10)$ and syringe $(\mathrm{n}=10)$ teaching methods. Participants were provided with written instructions for their allocated method (Figure 2) and afforded as much time as they deemed necessary to practise the method.

For the biofeedback method, participants were able to directly read the force values on the measuring scales when pressing on the airway model and therefore used real-time feedback to apply the recommended cricoid pressure force for "awake" and "anaesthetised" patients. For the nose method, participants were not given access to the airway model. They were given written instructions which advised that for "anaesthetised" patients a pressure on the nose to cause significant pain should be applied. Participants then practised pressing on their own nose to feel the force required. There were no teaching instructions given for "awake" patients, since this is not provided in the literature[7]. The syringe method consisted of a $50 \mathrm{ml}$ closed syringe enclosed in a wooden box with a $50 \mathrm{ml}$ syringe attached to the plunger (Figure 3). Compression of the enclosed syringe to $38 \mathrm{ml}$ is reported to be equivalent to the recommended pressure for "awake" patients 
and compression of enclosed syringe to $30 \mathrm{ml}$ is reported to be equivalent to the recommended pressure for "anaesthetised" patients[8].

\section{Post-teaching force measurement}

Participants were asked to repeat the blinded cricoid pressure force assessment using the same pharyngeal model and measuring scales 3 times in both "awake" and "anaesthetised" patients .

\section{Ethical considerations}

Participation in the study was voluntary. Informed verbal consent was obtained from participants prior to study commencement. Ethical approval was deemed not to be required by the Clinical Effectiveness Department of Sandwell and West Birmingham Hospitals NHS Trust. There were no additional risks or benefits for participants in the study.

\section{Confidentiality, data storage, security}

Measurements were recorded on paper forms which were stored securely in the City Hospital ICU, a restricted security access area. After data collection, the information on the paper forms was collated onto an Excel spreadsheet with each participant allocated a unique de-identified number; paper copies were then shredded. The electronic spreadsheet was stored securely on a National Health Service (NHS) computer and was file and folder password protected. Data was only accessible to, and used by, the study investigators in this study.

\section{Statistical analysis}

An overall model to assess differences in force according to the method of teaching was used. The dependent variable was the "post-teaching measurements", categorical variables indicated patient status ("awake" and "anaesthetised") and teaching method ("biofeedback", "nose" and "syringe"), and their interaction, and "baseline measurements" were considered as a continuous covariate. This model is conventionally formulated as an analysis of covariance (ANCOVA) and in Stata can be estimated using the "anova" (analysis of variance), "regress" (linear regression) or "mixed" (linear mixed [random effects] model, LMM)[21]. For the LMM, a random coefficient model was formulated with participants considered as random intercepts and baseline measurements as random effect slopes (for each participant). The incorporation of both "anaesthetised" and "awake" tests into the overall model allowed the parts of the model to borrow strength from each other. Model predictions were calculated using the "margins" command of Stata[22]. Model adequacy for the ANCOVA, linear regression and LMM models was adjudged according to the information criteria; lower scalar values preferred (AIC, assessing best model prediction), Bayesian information criterion (BIC, best overall model) and the likelihood ratio test[23]. Model estimates for each "method" and "status" are displayed in graph format using the Stata "marginsplot" command.

Exact P-values are reported for parameter estimates; P-values $<0.05$ were deemed to be significant. Statistical analysis was performed using Stata (V 15.1) software.

\section{RESULTS}

\section{Participant characteristics}

Thirty participants were recruited; 9 (30\%) anaesthetic/ICU junior and senior medical staff, $20(67 \%)$ Operating Department Practitioners, Assistant Theatre Practitioners and ICU nurses and $1(3 \%)$ student Operating Department Practitioner (Table 1). Seven (23\%) participants had experience in applying cricoid pressure 1-10 times, 11 (37\%) 10-100 times and 12 (40\%) over 100 times.

\section{Force measurement}

At baseline, the required force was applied in $9(30 \%)$ and $8(27 \%)$ participants for "awake" and "anaesthetised" patients respectively. Eight (27\%) participants applied less than the force required to prevent 
aspiration for "awake" patients and $18(60 \%)$ applied less than the required force for "anaesthetised" patients. Thirteen (43\%) participants applied forces greater than recommended for "awake" patients and 4 (13\%) participants applied greater than recommended force for "anaesthetised" patients (Figure 5).

The LMM (random coefficient) had the best model fit as adjudged by the information criteria for evaluating the difference in applied force according to the 3 different teaching methods (Table 3). Baseline cricoid pressure forces was a weak determinant of post-teaching cricoid pressure forces $(\mathrm{P}==0.053)$. LMM coefficient and difference in predicted force estimates for the biofeedback, nose and syringe methods in "awake" and "anaesthetised" patients are shown in Tables 3 and 4.

Model estimates and marginal predictions of post-teaching forces between the 3 teaching methods and in "awake" vs "anaesthetised" patients yielded considerable differences in applied cricoid pressure. Importantly, the biofeedback method led to predicted forces within the recommended limit for both "awake" and "anaesthetised" patients. Graphical representation of predicted force is displayed in Figure 6.

\section{DISCUSSION}

\section{Comparison with current literature}

The correct application of cricoid pressure is used to reduce the risk of aspiration during induction by compressing the oesophagus[1]. Importantly, despite all the study participants being clinically experienced in the application of cricoid pressure, $70 \%$ did not apply the correct pressure at baseline for either "awake" or "anaesthetised" patients. In particular, nearly half of all participants applied excessive force on the simulated "awake" patients which, clinically, can lead to pain and retching[9]. Moreover, the force applied to "anaesthetised" patients was less than recommended in the majority of participants. Ineffective pressure can increase the risk of aspiration[9]. Our results differ from previous studies which suggest that most assistants apply less cricoid pressure than is required during intubation[4,12-14]. There are several potential reasons for this including some studies using greater recommended force values for "anaesthetised" patients $(>40 \mathrm{~N}$ versus $>30 \mathrm{~N}$ in our study) [4,12], and differences in simulation airway models[13] and study populations (e.g. anaesthetic assistants versus medical specialists ) [4,12-14].

Following teaching, the biofeedback and syringe teaching methods resulted in similar applied pressure (syringe method $\sim 5 \mathrm{~N}$ higher) in "anaesthetised" patients. This finding is not surprising since the model estimates for both methods fell very close to, if not inside, the recommended range of 30-40N. However, in "awake' patients, the biofeedback method was more effective than the syringe method for teaching participants the required cricoid pressure force. The force applied using the syringe method was $\sim 9 \mathrm{~N}$ higher than the force applied using the biofeedback method. More importantly, the predicted syringe method force was higher than recommended for "awake" patients which, as noted above, can induce adverse clinical sequelae. Our findings differ to those of other studies which have reported that the syringe method leads to forces within the recommended range[8,20]; however, Flucker et al. trained and tested participants over a 1 month period which may contribute to the dissimilar findings [8]. Nonetheless, the results of our study confirm earlier reports that the biofeedback method is easy to teach and is the most effective technique for teaching the correct cricoid pressure[2,10,15-19].

Using the nose method to teach recommended pressure in "anaesthetised" patients yielded predicted cricoid pressure forces that were significantly lower $(\sim 9 \mathrm{~N})$ than those obtained using the biofeedback method. More importantly, the force applied was less than recommended which can lead to aspiration in patients undergoing intubation. [9]. Our findings are similar to the only other study that has assessed the nose method. The authors also reported this technique to be ineffective; albeit different force measurements were taken and target cricoid pressure forces for "anaesthetised" patients were 25-35 N, not the 30-40 N target pressure used in our study[7].

\section{Strengths}

Whilst comparison of the effectiveness of the common methods have been tested in other studies, most studies have only compared 2 methods $[2,7,10,15,17-19]$. Our study compares 3 different teaching methods 
with appropriate statistical contrasts. Furthermore, despite being widely recognised and used as a teaching method in clinical practice, only one study has tested the effectiveness of the nose method[7]. Our study sample was a good representation of staff routinely involved with cricoid pressure application from theatres and ICU and included a range of doctors and nursing anaesthetic assistants of different experiences and backgrounds across 2 different hospitals. Although the sample was opportunistic rather than random, we believe it is representative of those routinely performing cricoid pressure.

\section{Limitations}

First, the data was only collected at two sites on a single study day and a larger sample across more sites and over a longer period of time would increase the external validity and generalisability of the study results. Nonetheless, other investigators have reported that performance is variably maintained up to 3 months after teaching $[4,8,13]$. Second, the participants were sequentially allocated to the different teaching methods and random allocation would have improved the internal validity of the study. Third, the method of measuring applied pressure relied on the study investigators reading the unblinded real-time values on the measuring scales which may have contributed to bias in the study results. Fourth, the airway model is not a true representation of a patient and whilst the general anatomy and size are similar, the different texture, resistance and lack of overlying soft tissue and other attached structures (i.e. head and torso) limit real-life applicability. Of note, some investigators have used cadavers instead of airway models for assessing cricoid pressure[24]. Fifth, the height of the table on which the cricoid model was placed may not be the same as the height of an intubation table. Sixth, the varying experience of the participants was not accounted for. Finally, study conditions, such as the position of the participant when applying the force, were not protocolised.

The results of our study confirm earlier reports that the biofeedback method is easy to teach and is the most effective technique for teaching the correct cricoid pressure[2,10,15-19]. Whilst we did not assess performance over time, other investigators have reported that performance is variably between one week and 3 months $[4$, $8,13]$

\section{Conclusion}

Most participants that undertake cricoid pressure in their usual clinical practice did not apply forces within the recommended ranges in simulated awake and anaesthetised patients. However, recommended force can be achieved following biofeedback teaching. We recommend that formal teaching using this method be provided to all staff performing cricoid pressure to ensure the recommended pressure is applied.

\section{ACKNOWLEDGMENTS}

None

\section{COMPETING INTERESTS}

No external funding and no competing interests declared.

\section{REFERENCES}

1. Sellick BA. Cricoid pressure to control regurgitation of stomach contents during induction of anaesthesia. The Lancet 1961;278:404-6.

2. Owen H, Follows V, Reynolds KJ, Burgess G, Plummer J. Learning to apply effective cricoid pressure using a part task trainer. Anaesthesia 2002;57:1098-101.

3. Andruszkiewicz P, Zawadka M, Kosińska A, Walczak-Wieteska P, Majerowicz K. Measurement of cricoid pressure force during simulated Sellick's manoeuvre. Anaesthesiology intensive therapy 2017;49:283-7.

4. Ashurst N, Rout CC, Rocke DA, Gouws E. Use of a mechanical simulator for training in applying cricoid pressure. British journal of anaesthesia 1996;77:468-72. 
5. Clark RK, Trethewy CE. Assessment of cricoid pressure application by emergency department staff. Emergency medicine Australasia: EMA 2005;17:376-81.

6. Clayton TJ, Vanner RG. A novel method of measuring cricoid force*. Anaesthesia 2002;57:326-9.

7. Escott ME, Owen H, Strahan AD, Plummer JL. Cricoid pressure training: how useful are descriptions of force? Anaesthesia and intensive care 2003;31:388-91.

8. Flucker CJ, Hart E, Weisz M, Griffiths R, Ruth M. The 50-millilitre syringe as an inexpensive training aid in the application of cricoid pressure. European journal of anaesthesiology 2000;17:443-7.

9. Stewart JC, Bhananker S, Ramaiah R. Rapid-sequence intubation and cricoid pressure. International journal of critical illness and injury science 2014;4:42-9.

10. Komasawa N, Kido H, Minami T. Cricoid pressure force retention analysis using a simulator. British journal of anaesthesia 2016;117:405-6.

11. Parry A. Teaching anaesthetic nurses optimal force for effective cricoid pressure: a literature review. Nursing in critical care 2009;14:139-44.

12. Herman NL, Carter B, Van Decar TK. Cricoid pressure: teaching the recommended level. Anesthesia and analgesia 1996;83:859-63.

13. Cook TM, Godfrey I, Rockett M, Vanner RG. Cricoid pressure: which hand? Anaesthesia 2000;55:64853.

14. Koziol CA, Cuddeford JD, Moos DD. Assessing the force generated with application of cricoid pressure. AORN journal 2000;72:1018-28,1030.

15. Connor CW, Saffary R, Feliz E. Performance of the Sellick maneuver significantly improves when residents and trained nurses use a visually interactive guidance device in simulation.Physiological measurement 2013;34:1645-56.

16. Johnson RL, Cannon EK, Mantilla CB, Cook DA. Cricoid pressure training using simulation: a systematic review and meta-analysis. British journal of anaesthesia2013;111:338-46.

17. Kopka A, Crawford J. Cricoid pressure: a simple, yet effective biofeedback trainer. European journal of anaesthesiology 2004;21:443-7.

18. May P, Trethewy C. Practice makes perfect? Evaluation of cricoid pressure task training for use within the algorithm for rapid sequence induction in critical care.Emergency medicine Australasia: EMA 2007;19:207-12.

19. Quigley P, Jeffrey P. Cricoid pressure: assessment of performance and effect of training in emergency department staff.Emergency medicine Australasia: EMA 2007;19:218-22.

20. Kopka A, Robinson D. The $50 \mathrm{ml}$ syringe training aid should be utilized immediately before cricoid pressure application.European journal of emergency medicine: official journal of the European Society for Emergency Medicine 2005;12:155-8.

21. Huck SW, McLean RA. Using a repeated measures ANOVA to analyze the data from a pretest-posttest design: A potentially confusing task. Psychological bulletin 1975.

22. Williams R. Using the Margins Command to Estimate and Interpret Adjusted Predictions and Marginal Effects. The Stata journal 2012;12:308-31.

23. Kuha J. AIC and BIC: Comparisons of Assumptions and Performance. Sociological methods \&3 research 2004;33:188-229.

24. Vanner RG, Pryle BJ. Regurgitation and oesophageal rupture with cricoid pressure: a cadaver study. Anaesthesia 1992;47:732-5.

Table 1 Patient demographics. Values are n (\%).

Table 2 Comparison of ANCOVA, LMM and linear regression model.

Table 3 LMM coefficient estimates.

Table 4 The effect of methods and status on post-teaching values; estimates and differences are in newton units.

Figure 1 The airway model consisted of a pharyngeal model ${ }^{\mathrm{TM}}$ attached to measuring scales. 
Figure 2 Written instructions describing each method, which were provided to participants.

Figure 3 Syringe model used in study.

Figure 4 Overview of the study design.

Figure 5 Boxplot graph illustrating cricoid pressure force (N, mean) with $95 \%$ confidence intervals, applied at baseline (blue) and post-teaching (red) on "awake" and "anaesthetised" patients. Target ranges are shaded green.

Figure 6 Graph showing the model estimates (N, mean) with $95 \%$ confidence intervals of cricoid pressure post-teaching forces for each "method" and "status". Target ranges are shaded green.

\section{Hosted file}

Table 1.pdf available at https://authorea.com/users/370625/articles/489231-assessment-ofthe-most-effective-method-of-teaching-cricoid-pressure-force

\section{Hosted file}

Table 2.pdf available at https://authorea.com/users/370625/articles/489231-assessment-ofthe-most-effective-method-of-teaching-cricoid-pressure-force

\section{Hosted file}

Table 3.pdf available at https://authorea.com/users/370625/articles/489231-assessment-ofthe-most-effective-method-of-teaching-cricoid-pressure-force

\section{Hosted file}

Table 4.pdf available at https://authorea.com/users/370625/articles/489231-assessment-ofthe-most-effective-method-of-teaching-cricoid-pressure-force
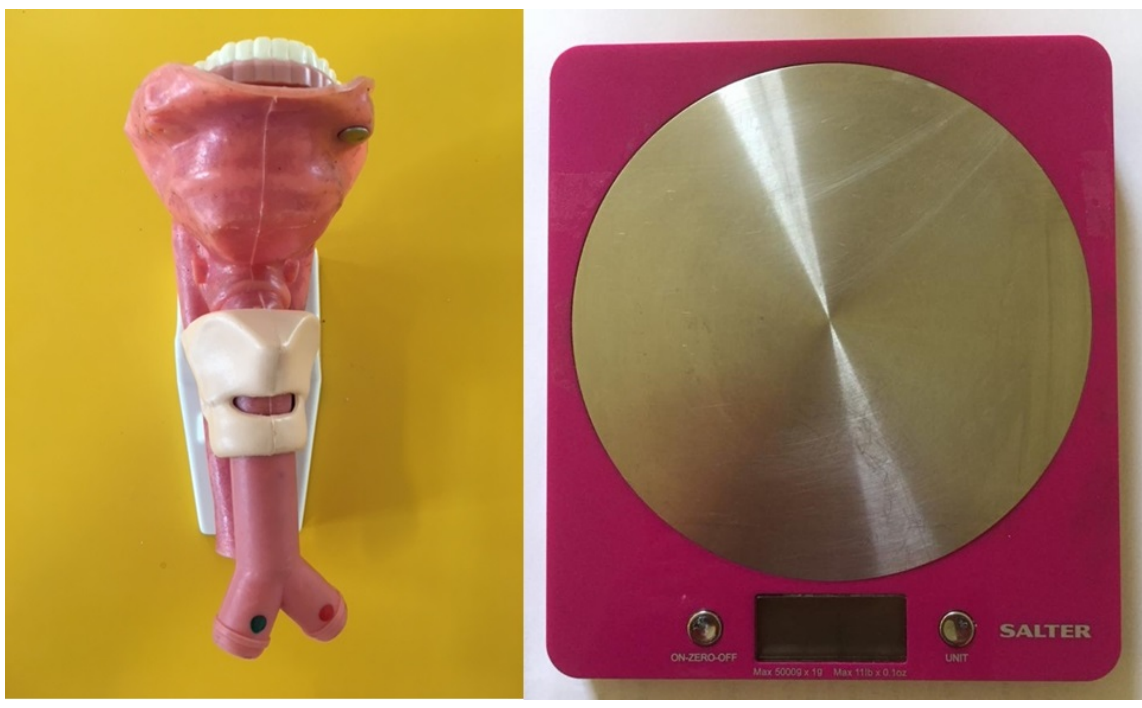


\section{Biofeedback method}

"Awake" cricoid pressure is $1-2 \mathrm{~kg}$

"Anaesthetised" cricoid pressure is $3-4 \mathrm{~kg}$

Practise on the model, applying these pressurevalues to the cricoid cartilage

Nose method

"Awake" - no advice

"Anaesthetised" - Use a pressure significant to cause pain if applied to the bridge of the nose

Syringe method

Apply pressure to the device to plunge the syringe to:

"Awake"-38ml

"Anaesthetised" - 30ml 


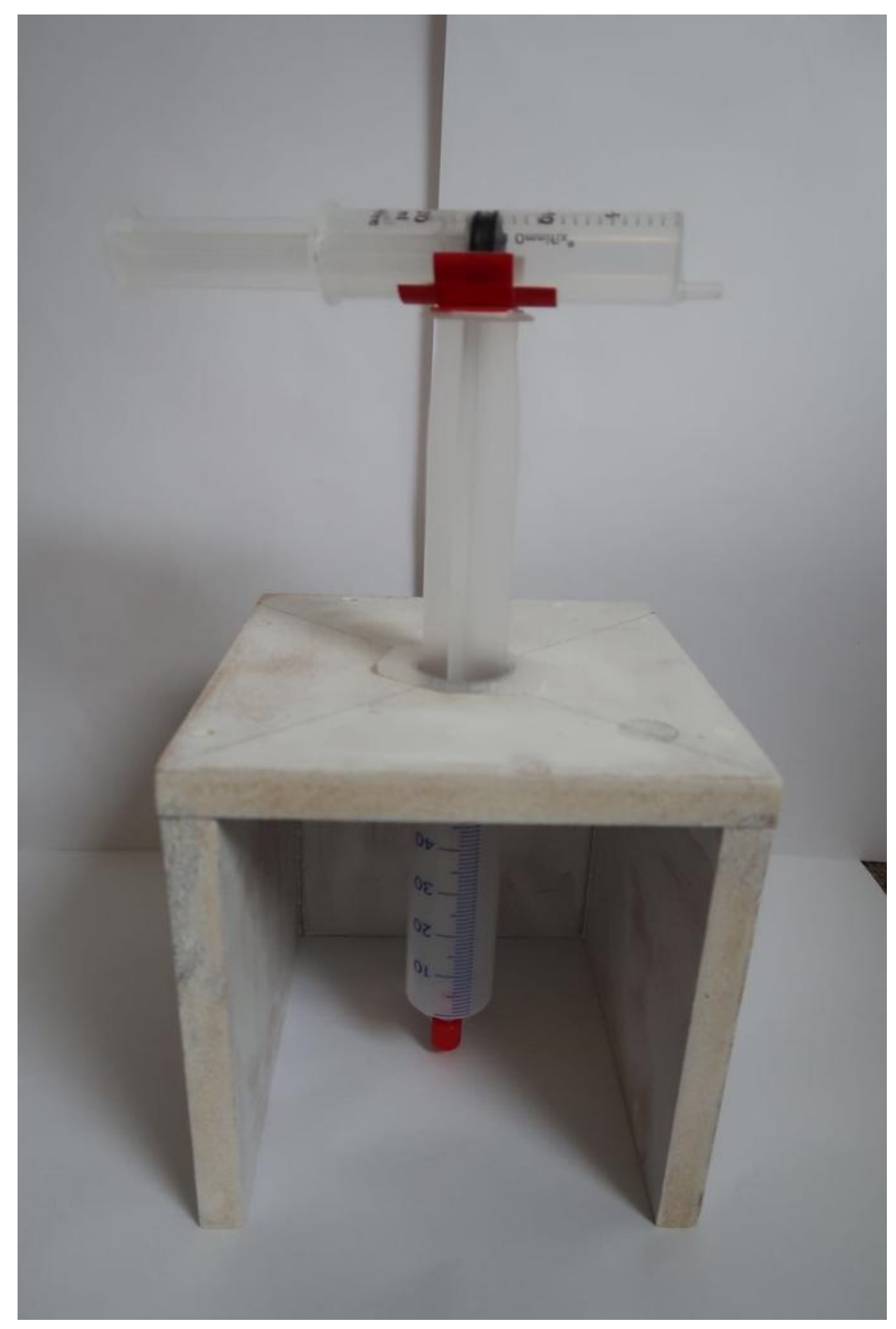


Recruitment ICU and OT doctors and nurses who assist or carry out RSI as part of their job role

and were able to participate on data collection day were recruited.

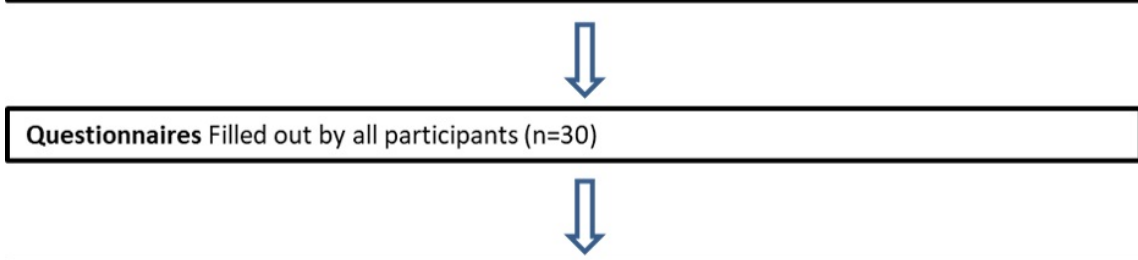

Baseline measurements Each participant applied cricoid pressure to the airway model x3 $(n=30)$

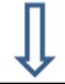

Intervention Each participant was allocated to one of three teaching methods and provided with written teaching instructions

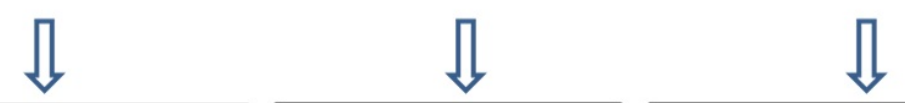

Biofeedback method $(n=10)$

Nose method $(n=10)$

Syringe method $(n=10)$

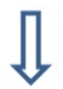

Post-teaching measurements Repeat measurements of cricoid pressure on the airway model $x 3$ $(n=30)$

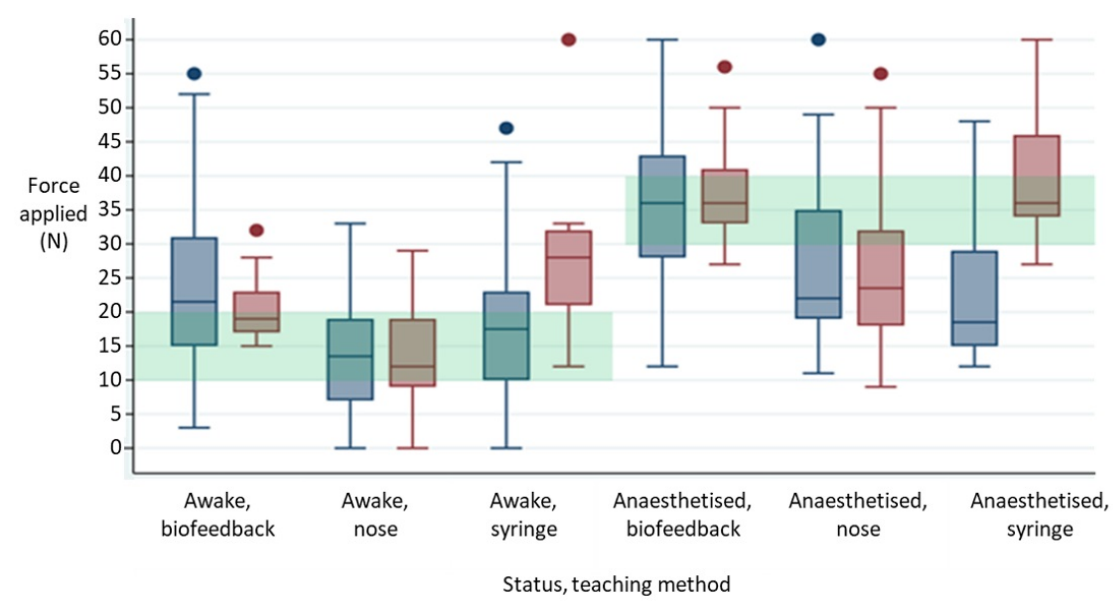




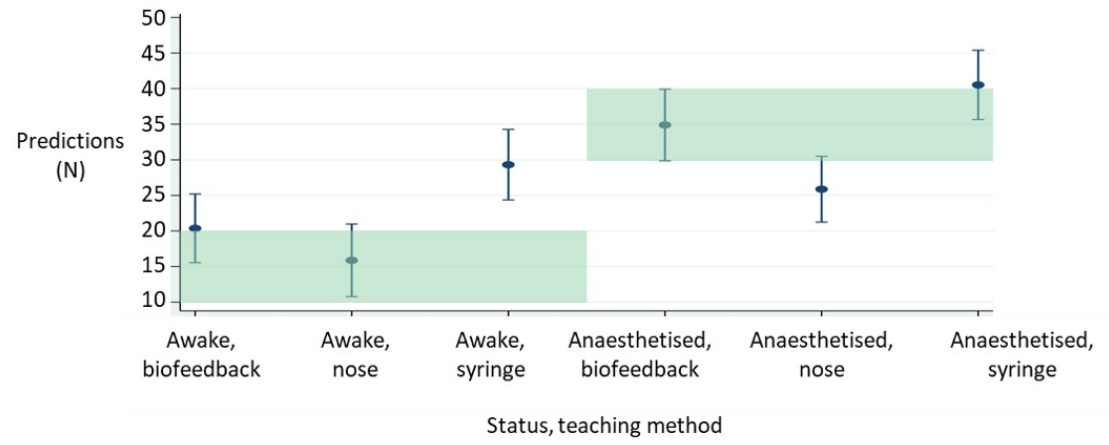

